\title{
Spontaneous Activity in Developing Ferret Visual Cortex In Vivo
}

\author{
Chiayu Chiu ${ }^{1}$ and Michael Weliky ${ }^{2}$ \\ 1/nterdepartmental Program in Neuroscience and ${ }^{2}$ Department of Brain and Cognitive Sciences, University of Rochester, \\ Rochester, New York 14627
}

\begin{abstract}
Multi-electrode extracellular recordings in area 17 of awake behaving ferrets were conducted to characterize the pattern of spontaneous activity in the developing visual cortex before eye opening. A linear array of 16 microwire electrodes was used to record extracellular neuronal activity across a $3.2 \mathrm{~mm}$ strip of visual cortex between postnatal days 22 and 28. Whereas synchronous bursts of activity were observed at all recording sites, cross-correlation analysis revealed that the timing of spike activity at all electrodes was not precisely correlated. Correlated activity between cortical sites exhibited a patchy organization having long-range components. Long-range cor-
\end{abstract}

Correlated patterns of spontaneous activity have been observed within early stages of the developing visual pathway, such as the retina and lateral geniculate nucleus (LGN). In vivo electrophysiological recordings in the retina of prenatal rats reveal significant correlations in the spontaneous discharge of neighboring ganglion cells (Maffei and Galli-Resta, 1990). In retinal slices of newborn ferrets and fetal cats, the spontaneous discharges form waves that propagate across the retina in a rhythmic manner (Meister et al., 1991; Wong et al., 1995). These retinal waves are relayed to the developing LGN, driving periodic trains of activity within the LGN in vitro (Mooney et al., 1996). Spontaneous bursting activity is observed in the developing LGN in vivo, exhibiting a highly specific laminar correlational structure (Weliky and Katz, 1999). Although correlated activity is highest in LGN layers that receive projections from the same eye, there are significant binocular correlations across different eye-specific layers. Synchronized bursting in the retina and LGN has been postulated to play an important role in the development of visual system circuitry before the onset of vision (Wong et al., 1993; Katz and Shatz, 1996). For example, the segregation of retinal ganglion afferents into eye-specific layers in the LGN is disrupted when spontaneous activity is blocked in the retina or LGN (Shatz and Stryker, 1988; Penn et al., 1998).

Beyond the retina and LGN, very little is known about the in vivo patterns of spontaneous activity, such as those in primary visual cortex. However, in vitro experiments in developing rat neocortical slices demonstrate the presence of spatiotemporally organized patterns of spontaneous cortical activity (Yuste et al., 1992; Schwartz et al., 1998). In these tangential sections, spontaneous intracellular calcium elevations are synchronized among

\footnotetext{
Received May 7, 2001; revised Aug. 31, 2001; accepted Sept. 4, 2001.

This work was supported by National Institutes of Health Grant EY12494 and the McKnight Foundation.

Correspondence should be addressed to Michael Weliky, Department of Brain and Cognitive Sciences, Meliora Hall, University of Rochester, Rochester, NY 14627. E-mail: weliky@cvs.rochester.edu.

Copyright (C) 2001 Society for Neuroscience $\quad 0270-6474 / 01 / 218906-09 \$ 15.00 / 0$
}

related activity was observed between cortical patches that were separated by a mean distance of $1 \mathrm{~mm}$. The spatial pattern of correlated activity persisted during transient lateral geniculate nucleus (LGN) activity block, indicating that longrange correlated activity is generated by intrinsic circuits within the cortex, independent of LGN input activity. These results demonstrate an innate patchy organization of correlated spontaneous activity within the cortex during the early development of cortical functional and anatomical organization.

Key words: extracellular recording; area 17; ferret; correlated activity; multi-electrode; visual cortical development neighboring cells so as to form discrete neuronal domains. In addition, functional synaptic circuits in layer 1 are detected in non-Cajal-Retzius neurons that share coincident spontaneous calcium transients.

Experimental evidence indicates that neuronal activity within the cortex itself is crucial for the development of functional and anatomical cortical organization. For example, continuous silencing of cortical action potentials with tetrodotoxin (TTX) blocks the maturation of orientation selectivity (Chapman and Stryker, 1993). Moreover, horizontal connections in the visual cortex fail to form clusters after intracortical TTX infusion (Ruthazer and Stryker, 1996). These functional and anatomical cortical structures are present before the onset of visual experience (Chapman et al., 1996; Durack and Katz, 1996; Ruthazer and Stryker, 1996), indicating that visual experience is not necessary for their initial formation. Rather, spontaneous activity in the visual cortex before eye opening may play a role in driving these processes.

In the present study, the in vivo patterns of spontaneous activity in the visual cortex are characterized. We observed synchronized bursts of activity in the visual cortex of ferrets between postnatal day 22 (P22) and P28. Significant long-range correlations in spontaneous activity are detected in animals of all ages, which persist in the absence of geniculate input activity.

\section{MATERIALS AND METHODS}

Eight male sable ferret kits, aged P22-P28, were used for extracellular multielectrode recordings (Marshall Farms, North Rose, NY). All procedures were approved by the University of Rochester Committee on Animal Research.

Electrode implant. Anesthesia was induced and maintained by inhalation of isoflurane $(0.5-2.0 \%)$ in a 2:1 nitrous oxide/oxygen mixture via a gas mask and placed in a stereotaxic holder. A section of skull $\sim 3 \times 5$ $\mathrm{mm}$ was drilled to expose the lateral occipital cortex back to the tentorium. The exposed dura was reflected. To ensure that the electrode array recorded activity exclusively from area 17, the array was aligned mediolaterally and positioned to record along the caudal bank of the posterior lateral gyrus. The multielectrode array, attached to the headset, was lowered so that all electrodes just touched the cortical surface. The skull 
opening was covered with agar to immobilize the cortex, and the headset was affixed to the skull using dental acrylic.

Optic nerve transection. Animals were anesthetized by freely breathing a mixture of 2:1 nitrous oxide/oxygen, supplemented by $0.5-2.0 \%$ isoflurane. An incision was made along the top eyelid. The connective tissue and muscles attaching the eyeball to the orbit were cut away. Fine scissors were used to reach behind the eyeball to cut the optic nerve. The rear portion of the eyeball was rotated into view to visually confirm complete optic nerve transection. Finally, the skin along the incision was sutured. This procedure was performed for both eyes. Animals fully recover from anesthesia within 5-10 min after surgery.

Recording and data acquisition. The multielectrode array used for multiunit recording in the visual cortex consisted of a single row of 16 electrodes spaced $200 \mu \mathrm{m}$ apart. Electrodes were $2 \mathrm{~mm}$ in length and were made from 12.5- $\mu \mathrm{m}$-diameter tungsten wires with $2.5 \mu \mathrm{m} \mathrm{H}$-ML insulation (California Fine Wire, Grover Beach, CA). The electrode tips were made by shaving 30-40 $\mu \mathrm{m}$ of insulation off with a razor. The electrode array was glued to a shaft of $50-\mu \mathrm{m}$-thick stainless steel sheet, which was in turn affixed to a small plate within the metal headset. The plate could be moved up and down by turning a 100 thread per inch screw, thereby raising or lowering all electrodes simultaneously.

We determined whether distances between electrodes remained constant as the recording array was lowered into the cortex. This was done by pushing electrode arrays into freshly dissected cortical tissue that was cut into 1 - to $1.5-\mathrm{mm}$-thick blocks and placed on agar. We found that the spacing of electrodes in arrays was identical as they entered and exited the block of tissue, indicating that the electrodes did not shift positions in the tissue. In addition, the length of each microwire electrode is only 2 $\mathrm{mm}$. At this length, the wires are quite stiff and rigid. Thus, we can rule out the possibility that electrodes in the array significantly move out of their original alignment in the cortex.

During recording, animals were free to move within a low-walled small box placed on a heating blanket. All recordings of spontaneous activity were performed in a dark and acoustically insulated room. Eighteeninch-long small-diameter low-noise coaxial cables connected the animal's headset to custom-made amplifiers, providing 20,000 gain. Two stage resistor-capacitor circuits band-pass filtered the signal between 600 and $6000 \mathrm{~Hz}$. The amplifiers were mounted above the animal to provide freedom of movement within the box. The amplifier output was fed into a personal computer plug-in analog-to-digital board (National Instruments, Austin, TX) and digitized at $10 \mathrm{kHz}$. The acquisition was controlled through custom software written in LABVIEW (National Instruments, Austin, TX). Spikes were discriminated from noise based on voltage threshold, which was set at 4.3 times the SD of noise.

Recording sessions lasted between 14 and $24 \mathrm{hr}$, during which animals were resting quietly $\sim 90 \%$ of the time. Each session was divided into 5-10 recording blocks, each in turn was composed of $26100 \mathrm{sec}$ recording trials. The interval between successive $100 \mathrm{sec}$ recording trials within a block was typically $\sim 5 \mathrm{sec}$.

Measurements and calculations. Identification of a macroburst was performed by computing a moving window average (width, $1000 \mathrm{msec}$ ) of discriminated spike activity across all 16 electrodes in $100 \mathrm{sec}$ recording trials. A threshold was established to remove low-amplitude background spike activity. The duration of a macroburst was calculated by determining the amount of time during which values were continuously above threshold.

Identification of microbursts at individual electrodes was performed by computing a Gaussian weighted moving window average (width, 15 msec) of discriminated spike activity. A threshold was established to remove low-amplitude background activity. The duration of a microburst was determined by calculating the period of time during which the weighted average was continuously above threshold.

The mean distance between correlation peaks was calculated for each animal by only including cases when secondary peaks showed a rising and falling phase to either side of the peaks. Secondary peaks were not included in the calculation when they occurred at the extreme right or left of correlation maps. The values from all animals were then averaged to obtain the mean distance between peaks.

Cross-correlation function calculations. The cross-correlation function between spike trains recorded at two electrodes was calculated by computing all time intervals between a spike at one electrode and a spike at the second electrode during one or more $100 \mathrm{sec}$ recording trials, binned into successive time bins. The result was divided by the total number of spikes from the first electrode and the bin width. This yielded a histogram of the spike firing rate at electrode two as a function of time since a spike from electrode one. This result was normalized by dividing by the total number of spikes from the second electrode and multiplied by a scale factor of 100 .

Statistical analysis. The Pearson product correlation coefficient $r$, defined by

$$
r=\frac{\Sigma(X-\bar{X})(Y-\bar{Y})}{\sqrt{\Sigma(X-\bar{X})^{2} \Sigma(Y-\bar{Y})^{2}}}
$$

describes the strength of the association between two variables. Values of $r$ range from -1.0 to 1.0 , although analysis of our data yielded only positive values because of the exclusively positive correlations in activity. Points in $X$ represent binned spike counts (bin size ranging from 4 to 260 $\mathrm{msec}$ ) at successive time points (time interval equals the bin size) for one electrode. Points in $Y$ represent the corresponding binned spike counts at a second electrode. The correlation coefficient $r$ was calculated between different electrode pairs from a single discontinuous $43.3 \mathrm{~min}$ time series plot of spike data collected over one recording block. The resulting correlation values we obtain are a direct measurement of $r$ between different cortical sites. This cross-correlation coefficient provides a normalized measurement of the covariance of spike firing rates independent of levels of neuronal activity.

To determine whether the computed correlation coefficients were the result of random fluctuations in activity, the $t$ test having the following form

$$
t=\frac{r}{\sqrt{\left(1-r^{2}\right) /(n-2)}}
$$

was used to assess their statistical significance. $r$ is the correlation value with $n-2$ degrees of freedom. A $p$ value of 0.05 was used to determine significance level. No multiple comparisons were made here.

The statistical significance of secondary peaks was determined for each cross-correlation map by assessing the significance of the difference between two correlation coefficients $r$ (Snedecor and Cochran, 1989). Specifically, the $r$ value at the baseline trough $(T)$ and at the primary or secondary peak $(P)$ was each converted to $z$ values, using $z=(1 / 2)$ $[\ln (1+r)-\ln (1-r)]$. Then, the difference between the $z$ values was calculated $\left(D=z_{P}-z_{T}\right)$. The variance of $D$ was obtained by adding the individual variances of each $z$ value

$$
\left(\sigma=\frac{1}{\sqrt{n-3}}\right) .
$$

Next, the normalized $z$ was calculated by dividing $D$ by $\sigma_{D}$. Significance of the difference was determined with the aid of Snedecor and Cochran's (1989) Table A4 for two-tailed tests in the appendix of Statistical Meth$o d s$, at the 0.05 significance level.

\section{RESULTS}

\section{Spontaneous bursts of neural activity}

Spontaneous neuronal activity was recorded across a $3.2 \mathrm{~mm}$ strip of primary visual cortex (area 17) in awake behaving ferrets (P22-P28) before natural eye opening. Multiunit recordings were obtained with a linear multielectrode array, consisting of a single row of 16 electrodes with $200 \mu \mathrm{m}$ spacing. At all ages, times series graphs demonstrate a waxing and waning of neuronal activity across all electrodes (Figs. 1a, 2a). The average duration of the periods of simultaneous activity across the electrode array, which we called macrobursts, was $16.1 \pm 5.1 \mathrm{sec}$ and the average frequency was 2.4 macrobursts/min. In all animals, these macrobursts did not result from a general elevation in spike firing rate, but instead cell firing at each electrode during a macroburst occurred in the form of elementary burst packets (microbursts) (Fig. 1b). These microbursts varied in duration from 50 to 150 msec and either occurred alone (inset 2 ) or occurred rhythmically at $\sim 10 \mathrm{~Hz}$ (inset 1). Raw spike traces at multiple electrode positions further revealed that activity during a macroburst was not precisely correlated at all electrodes (Fig. $2 b$ ). Temporal 


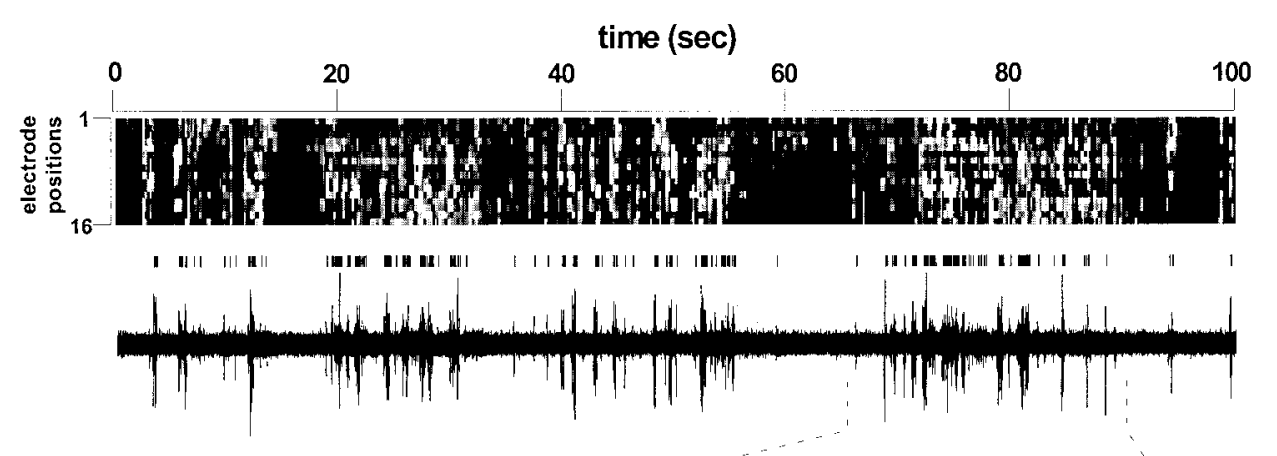

Figure 1. Synchronous neuronal activity in the visual cortex of neonatal awake behaving ferrets occurs as burst packets. $a$, Top trace, Time series graph was computed from a single $100 \mathrm{sec}$ acquisition trial for a P27 animal. It shows periodic macrobursts of neuronal activity across the 16 channel electrode array. Spike discharge rate at each electrode is encoded in gray scale along a different horizontal row (electrode 1 is the topmost row, and electrodes 2-16 are successive rows down). Bin width, $40 \mathrm{msec}$. Bottom trace, Spike activity in electrode 10 for the same recording trial. Note that the bursts in electrode 10 correspond in time to the macrobursts in the time series graph above. $b$, Close-up of a burst in electrode 10 reveals elemental burst components, which can either occur rhythmically (inset 1) or as a single microburst (inset 2). Microburst duration ranged from 50 to $150 \mathrm{msec}$. The vertical line segments above the traces indicate discriminated spikes.

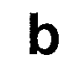

b
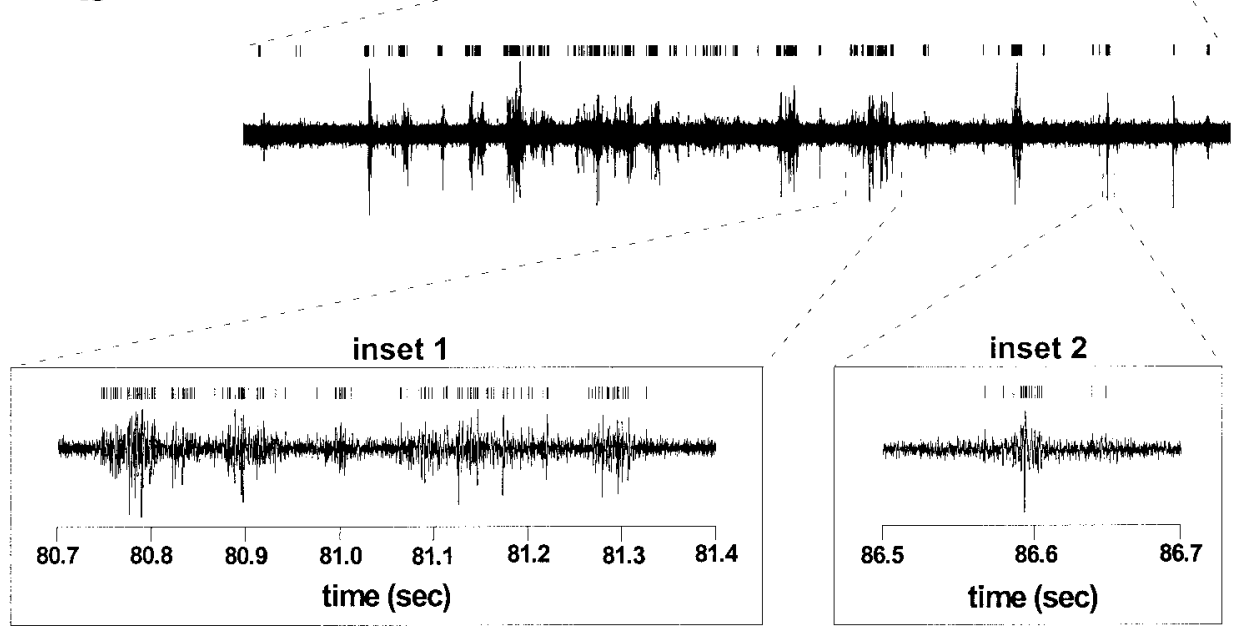

firing patterns within a macroburst typically varied from electrode to electrode.

\section{Cross-correlation analysis of neuronal activity}

To quantitatively assess systematic differences in correlated firing between different recording sites, cross-correlation maps were generated by computing the Pearson product correlation coefficient $(r)$ between spike trains recorded at each recording site and all other sites (Figs. 2c, 3a) (see Fig. 7). Each cross-correlation map was constructed from a single discontinuous 43.3 min time series plot of collected spike data (see Materials and Methods). Cross-correlation maps in all eight animals revealed a long-range spatial pattern of correlated activity (Fig. 3). Recordings of spontaneous activity yielded cross-correlation maps that contained a primary peak of $r=1.0$, corresponding to the autocorrelation value of activity at the recording site. Correlated activity decreased as a function of distance away from the recording site and reached minimum values at a mean distance of $600 \pm 75 \mu \mathrm{m}$. However, a secondary peak of correlated activity, ranging from $r=0.11$ to 0.73 , could be observed in all animals. The mean distance between primary and secondary peaks was $1056 \pm 360$ $\mu \mathrm{m}$. For each cross-correlation map, the difference between baseline correlation coefficients and peak values was calculated and found to be statistically significant $(p<0.001$, modified twotailed $z$ test; see Materials and Methods). This long-range spatial pattern was not only observed in 43.3 min recording blocks but also in single $100 \mathrm{sec}$ trials, each of which consisted of only two to three macrobursts (Fig. 2c). In all cases, the positions of secondary peaks were identical from trial to trial and from block to block within the same recording session. For three of eight animals, cross-correlation analysis revealed multiple secondary peaks (Fig. $3 a, 1,3,7)$. The mean distance between the primary peak and the proximal and distal secondary peaks in these animals was $1112 \pm$ 365 and $2114 \pm 103 \mu \mathrm{m}$, respectively.

Previous work has suggested that different cortical layers exhibit different spontaneous activity properties (Miller, 1996). Because of the slight curvature of area 17 relative to our electrode tips, which were aligned along a straight line, it is possible that different electrodes may lie within different lamina. If laminar differences contributed to the patchy distribution of correlated activity, then altering the recording depth should result in a corresponding shift in the position of secondary peaks in crosscorrelation maps. Therefore, to examine the influence of laminar differences in spontaneous activity on the observed patchy distribution of correlation, we analyzed activity patterns at varying cortical depths between 250 and $900 \mu \mathrm{m}$. Patterns of correlated activity were not altered by changes in the recording depth of the electrode array (Fig. 2c). This observation indicates that the patches of correlated spontaneous activity span across cortical layers in a columnar manner.

\section{Analysis of spike timing}

To investigate how the timing of spike activity at different recording sites could underlie the generation of this correlation map, cross-correlation functions were calculated between all pairs of electrodes (Fig. 4). The normalized amplitudes of these functions were consistent with the computed Pearson product correlation coefficients. Whereas the discharge of spikes in neighboring cor- 
a
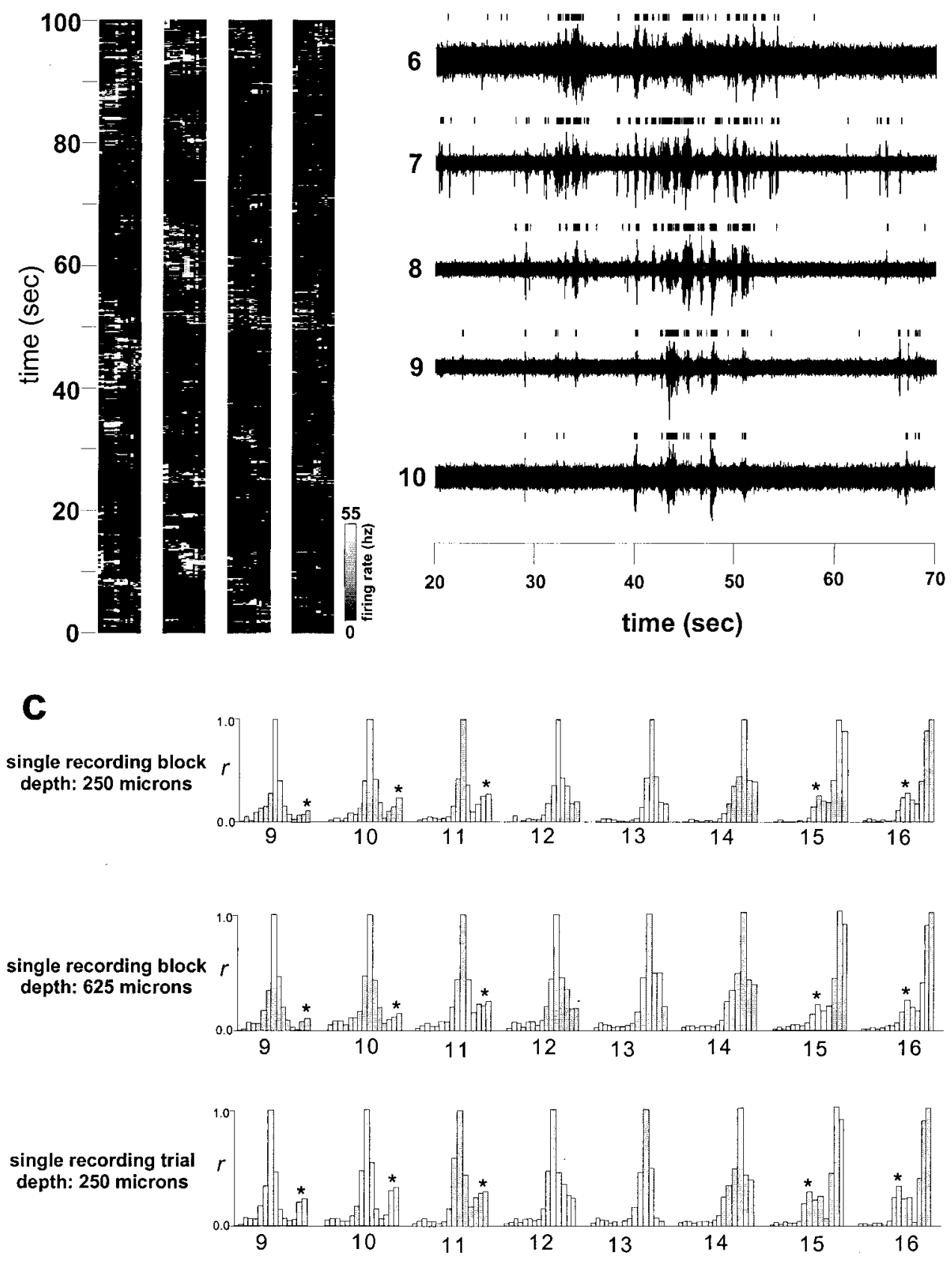

Figure 2. Cross-correlation analysis reveals long-range spatial organization. $a$, Four time series graphs shown for a P22 animal. Bin width, $40 \mathrm{msec}$. $b$, Spike activity in electrodes $6-10$ for the leftmost time series graph in $a$, from $20-70 \mathrm{sec}$, are shown. Although a macroburst was observed beginning at $33 \mathrm{sec}$, spikes were not precisely synchronized in time at all electrodes. Spike amplitudes typically vary from 50 to $150 \mu \mathrm{V}$. The vertical line segments above the traces represent discriminated spikes. $c$, Spatial pattern of correlated activity is stable at varying recording depths and can be observed in single trials. Cross-correlation maps obtained from the same P22 ferret are shown for eight different electrodes (electrodes 9-16). In each map, vertical bars plot the cross-correlation coefficient $(r)$ computed between spikes recorded at the labeled electrode and all other electrodes (electrode 2 is the left bar, and electrodes 3-16 are successive bars to the right). Significant long-range secondary peaks are marked by asterisks (modified $z$ test; $p<$ 0.001). Top trace, Cross-correlation map for a single 43.3 min recording block (26 successive trials) at a recording depth of $\sim 250 \mu \mathrm{m}$. Note the secondary peaks in correlation maps for electrodes 9, 10, 11, 15, and 16. Middle trace, Cross-correlation map for another recording block in the same animal after electrodes were moved to a depth of $625 \mu \mathrm{m}$. The secondary peaks are at identical positions when compared with the top trace. Bottom trace, Cross-correlation map from a single 100 $\mathrm{sec}$ recording trial obtained from the recording block shown in the top trace. Secondary peaks are present at identical positions. Bin width, $40 \mathrm{msec}$. tical sites was synchronized precisely in time (column 1), spike timing between more distant sites having a low correlation coefficient exhibited a variety of complex dynamics. Cross-correlation functions computed from different $100 \mathrm{sec}$ recording trials in the same animal revealed out-of-phase oscillations (column 2, trial 3), single peaks at nonzero phase positions (column 2, trial 2), and randomly timed cell activity (column 2, trial 1). Spike timing at the secondary peak in the correlation map once again occurred with zero time lag relative to the reference electrode (column 3). This complexity in dynamics is not surprising considering the wide temporal range of cell activity observed from single to rhythmic microbursts. Thus, differences in spike timing directly underlie the long-range correlation pattern we observe.

\section{Transiently blocking LGN activity by optic nerve transection: effects on the pattern of spontaneous activity}

Next, to explore mechanisms regulating spatial patterns of correlated spontaneous activity, we considered LGN inputs to the visual cortex. Recordings from our P26-P28 animals were compared with previous LGN recordings in ferrets of equivalent ages (P26-P28; $n=3$ ) (data from Weliky and Katz, 1999). The duration and frequency of macrobursts within the LGN and visual cortex were indistinguishable. The mean duration of macrobursts in the LGN and cortex was $14.8 \pm 7.5$ and $14.9 \pm 6.4 \mathrm{sec}$, respectively ( $p>0.05$; two-tailed $t$ test), and the mean frequency was 2.4 and 2.5 macrobursts/min, respectively $(p>0.05$; two- 
a

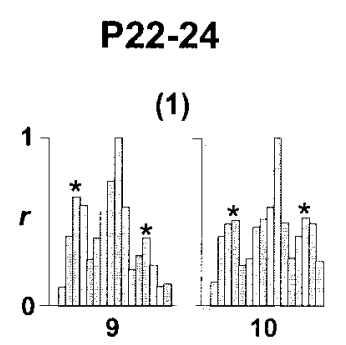

(2)

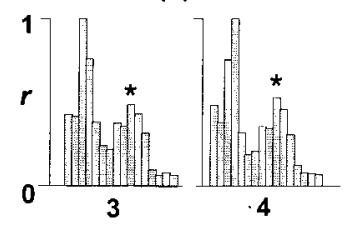

(3)

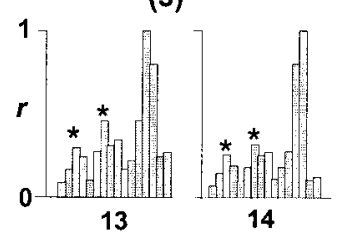

(4)

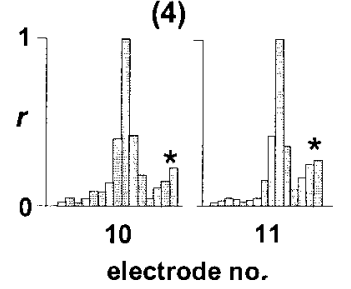

electrode no.

b

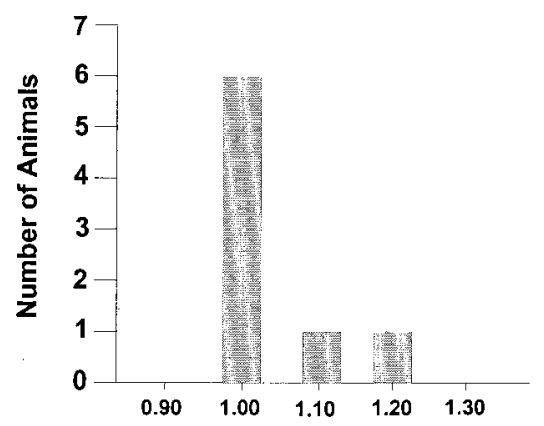

Avg Distance Between Peaks (mm)

Figure 3. Long-range cortical organization is present in all animals. $a$, Cross-correlation maps for two adjacent electrodes are depicted for each animal. Left, Maps containing secondary peaks for four P22-P24 animals; right, examples for four P26-P28 animals. Asterisks indicate significant secondary peaks. Note that three animals show additional peaks that are spaced $\sim 2 \mathrm{~mm}$ apart. $b$, Summary graph of the distribution of average distances between correlation peaks among all eight animals. A total of six animals exhibit an average peak spacing of $1 \mathrm{~mm}$.

tailed $t$ test). Although we did not perform simultaneous recordings in the LGN and visual cortex, this finding suggests that bursting within the LGN and visual cortex is likely synchronized through feedforward and feedback connections within the corticothalamic loop.

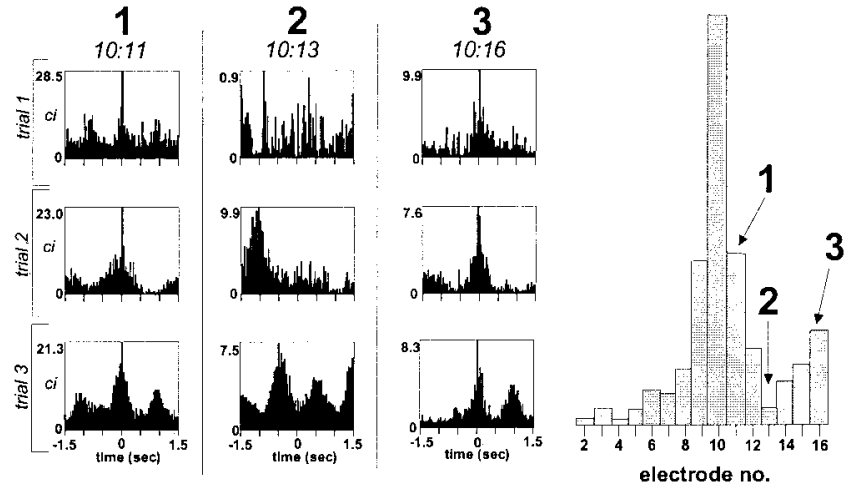

Figure 4. Timing of spike activity underlies long-range correlations. Cross-correlation functions calculated between pairs of electrodes separated by different distances in a P22 ferret. Each column shows correlation functions computed between spikes recorded at electrode 10 and electrodes 11,13 , and 16 respectively. The three rows show correlation functions calculated during different $100 \mathrm{sec}$ recording trials. 1, 2, and 3 denote the corresponding sites in the cross-correlation map on the right at which these functions were computed. Bin width, $20 \mathrm{msec}$.

To investigate how removal of LGN activity affects the pattern of visual cortical bursting, we used the finding that transection of both optic nerves abolishes all LGN activity for $\sim 50 \mathrm{~min}$, after which LGN activity gradually returns over a period of $6 \mathrm{hr}$, consisting of much more sharply defined macrobursts with shorter duration and increased frequency (Weliky and Katz, 1999, their Fig. 4b). For all animals at all ages, cutting both optic nerves immediately resulted in a sharpening of macrobursts within the visual cortex (Fig. 5). Macroburst duration in younger animals $(\mathrm{P} 22-\mathrm{P} 24 ; n=4)$ decreased from $17.3 \pm 3.9 \mathrm{sec}$ before optic nerve transection to $3.0 \pm 1.1 \mathrm{sec}$ after optic nerve transection $(p<0.05$; two-tailed $t$ test) and in older animals (P26-28; $n=4)$ from $14.9 \pm 6.4 \mathrm{sec}$ before optic nerve transection to $1.9 \pm 0.8 \mathrm{sec}$ after optic nerve transection ( $p<0.05$; two-tailed $t$ test). In addition, a significant increase in macroburst frequency was observed in older animals after optic nerve transection, from 2.5 to 5.5 macrobursts/min ( $p<0.05$; two-tailed $t$ test). In younger animals, the mean macroburst frequency before ( 2.2 macrobursts/ min) and after (2.6 macrobursts/min) optic nerve transection was not statistically different ( $p>0.05$; two-tailed $t$ test). Changes in cortical bursting remained constant for up to $16 \mathrm{hr}$, the longest period of recording, despite the recovery of stable LGN input activity after $6 \mathrm{hr}$. Moreover, in older animals (P26-P28), the duration and frequency of visual cortical activity was indistinguishable from the duration and frequency of LGN bursting in equivalent age animals after full recovery (data from Weliky and Katz, 1999). Six hours after optic nerve transection, the mean macroburst duration in the LGN and cortex was 1.8 and $1.9 \mathrm{sec}$, respectively, and the mean macroburst frequency in the LGN and cortex was 6.5 and 5.5 macrobursts/min, respectively $(p>0.05$; two-tailed $t$ test). This suggests that the pattern of LGN spontaneous activity that gradually emerges during the $6 \mathrm{hr}$ after optic nerve transection reflects the pattern of spontaneous activity already present in the visual cortex immediately after transection of both optic nerves. This observation, coupled with the finding that removal of visual cortical feedback to the LGN completely and permanently abolished LGN activity in bilaterally enucleated animals (Weliky and Katz, 1999), suggests that, at least after binocular enucleation, spontaneous activity within the thalamocortical loop is initially established in the visual cortex. 

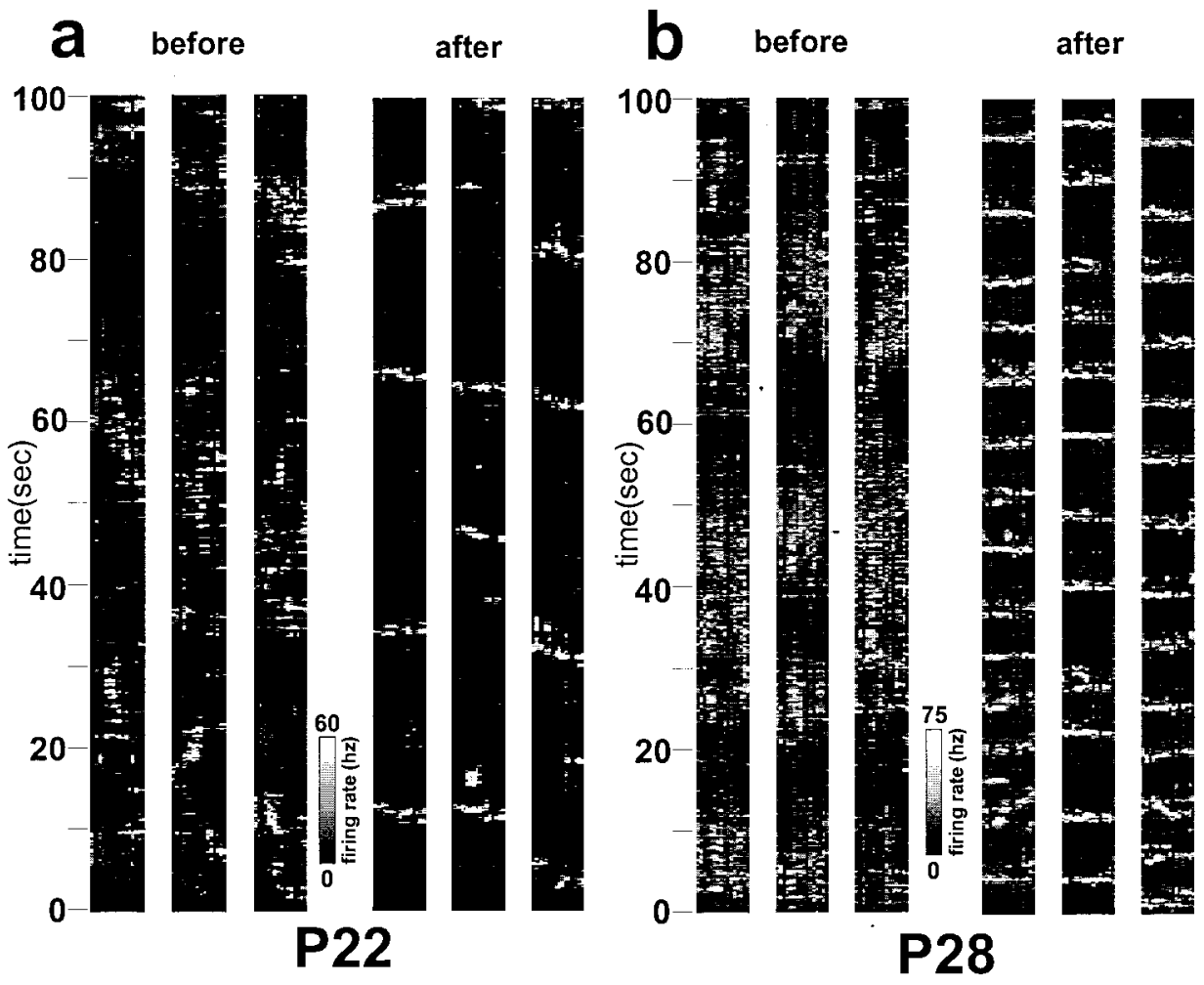

Figure 5. Optic nerve transection alters temporal patterns of visual cortical spontaneous activity. $a$, Time series graphs of visual cortical activity obtained from a P22 ferret. The first set of three graphs shows control activity with intact LGN activity. The second set of graphs shows changes in the pattern of visual cortical bursts after optic nerve transection, which completely abolishes LGN activity for approximately the first $50 \mathrm{~min}$ but stabilizes to a new spatiotemporal pattern over the following $6 \mathrm{hr}$. The first, second, and third graphs were obtained $10 \mathrm{~min}, 6 \mathrm{hr}$, and $13 \mathrm{hr}$, respectively, after optic nerve transection. There is no significant change in the visual cortical bursting pattern over this time period. $b$, Time series graphs of visual cortical activity obtained from a P28 ferret. Time series graphs are shown before and after cessation of LGN activity as in $a$. In the second set of graphs, the first, second, and third graphs were obtained $15 \mathrm{~min}, 5 \mathrm{hr}$, and $15 \mathrm{hr}$, respectively, after transection of both optic nerves. $c$, Left, Average duration of macrobursts of younger (P22-P24) and older (P26P28) animals before (white) and after (gray) transection of both optic nerves. At all ages, there was a significant decrease in macroburst duration, marked by asterisks. Error bars represent the SD from the mean. Right, Average frequency of macrobursts before and after optic nerve cut. Note that there is a significant increase in macroburst frequency only in P26-P28 animals.

Whereas the duration and frequency of macrobursts in the visual cortex were substantially altered after optic nerve transection in all animals, spiking activity at individual electrodes during macrobursts continued to be temporally organized as elementary microbursts (Fig. 6). Microbursts were still observed both alone and rhythmically. Additionally, the spatial pattern of long-range correlations was also unchanged after transection of both optic nerves in all animals at all ages (Fig. 7). Secondary peaks in each cross-correlation map were observed in exactly the same position before and after cutting both optic nerves for all animals, indicating that the cortex is capable of generating this spatial pattern of correlated activity independent of LGN input activity.

\section{DISCUSSION}

We demonstrate the presence of spontaneous rhythmic activity in the developing visual cortex in vivo. With intact LGN input drive, the temporal properties of cortical activity are similar to those of previously reported spontaneous oscillatory activity in the LGN, reinforcing the idea that LGN bursts are relayed to the cortex. However, our results further reveal a patchy distribution of correlated spontaneous activity in the visual cortex. Cortical regions that are separated by $\sim 1 \mathrm{~mm}$ exhibit significantly correlated oscillatory activity. The pattern of coincident activity is resistant to blockade of LGN input activity, indicating that the cortex can generate this pattern independently of the LGN.

Two potential mechanisms may underlie the patchy distribution of correlated activity in visual cortex. One possibility is the already segregated LGN axonal projections to the cortex representing the two eyes, observed as early as P16 in ferrets (Crowley and Katz, 2000). The other alternative is the long-range clustered horizontal connections in the cortex, which are first detected a few days after the observation of long-range correlated activity in P22 ferrets (Durack and Katz, 1996; Ruthazer and Stryker, 1996). 


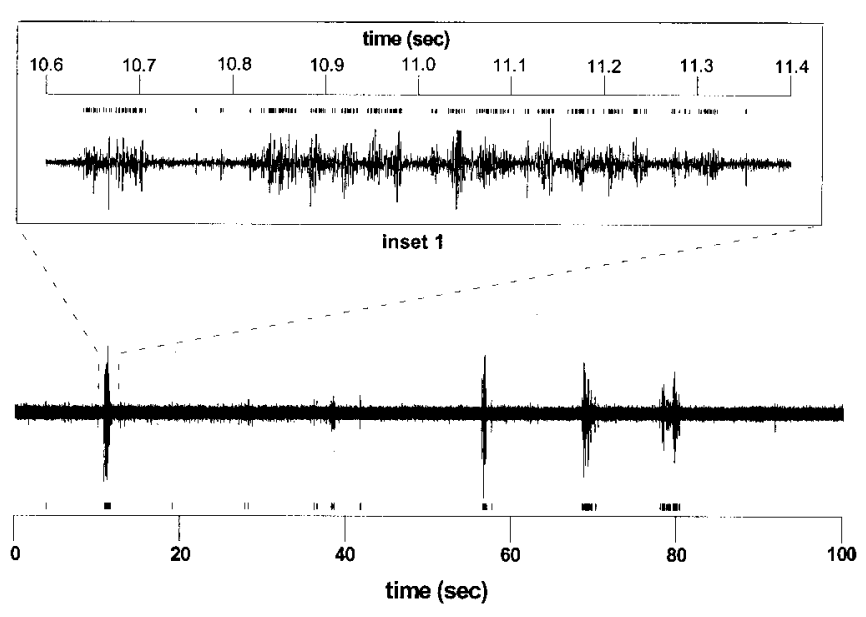

Figure 6. Single and rhythmic microbursts persist despite the absence of LGN input activity. Spike trace of activity acquired from electrode 16 of a P26 animal for a single trial 11.7 min after transection of both optic nerves. Inset 1, Close-up of a burst in electrode 16 revealed microbursts that occur alone and rhythmically. The vertical line segments above or below the traces represent discriminated spikes.

\section{LGN contributions to the pattern of correlated cortical activity}

One mechanism that may underlie long-range correlations in spontaneous activity is the segregated patches of diverging afferents from different eye-specific LGN layers (Crowley and Katz, 2000). These patches are first observed at P16, which is $6 \mathrm{~d}$ before our earliest recording at P22. Activity within the same eye-specific LGN layer, which has been shown to be more correlated than across different eye-specific layers (Weliky and Katz, 1999), may be relayed to distinct cortical patches via its axonal projections, thereby correlating activity in these patches. In this way, consistent with the work of Crowley and Katz (2000), the patches of correlated activity we observe would reflect the early development of ocular dominance columns. Our finding that the clustered pattern of correlated activity extends radially across cortical layers in a columnar manner reinforces this hypothesis. However, the same cortical patches continue to exhibit correlated activity in the absence of LGN input activity, indicating the presence of intrinsic cortical circuits that can generate this spatial pattern of activity independently of the LGN. It is possible that interactions between segregated LGN inputs and target cortical neurons could guide the establishment of modular cortical circuitry underlying this patchy pattern of correlated activity. Caríc and Price (1999) provide evidence that thalamocortical projections are important for the development of intrinsic cortical circuitry. They found that unilateral LGN lesion in newborn kittens disrupts corticocortical connections between area 17 and 18. It remains to be seen whether preventing the LGN afferents from innervating the cortex in the first place interferes with intrinsic circuit formation within area 17.

Although the average spacing between patches of correlated activity of $1 \mathrm{~mm}$ is different from the average width of ocular dominance columns of $\sim 600 \mu \mathrm{m}$ obtained by Crowley and Katz (1999), the significance of this difference is unclear. Unlike those in primates and cats, the organization of ocular dominance bands in area 17 of the ferret is highly variable in shape and size depending on regional location (White et al., 1999). Whereas ocular dominance columns at the posterior tentorial portion tend
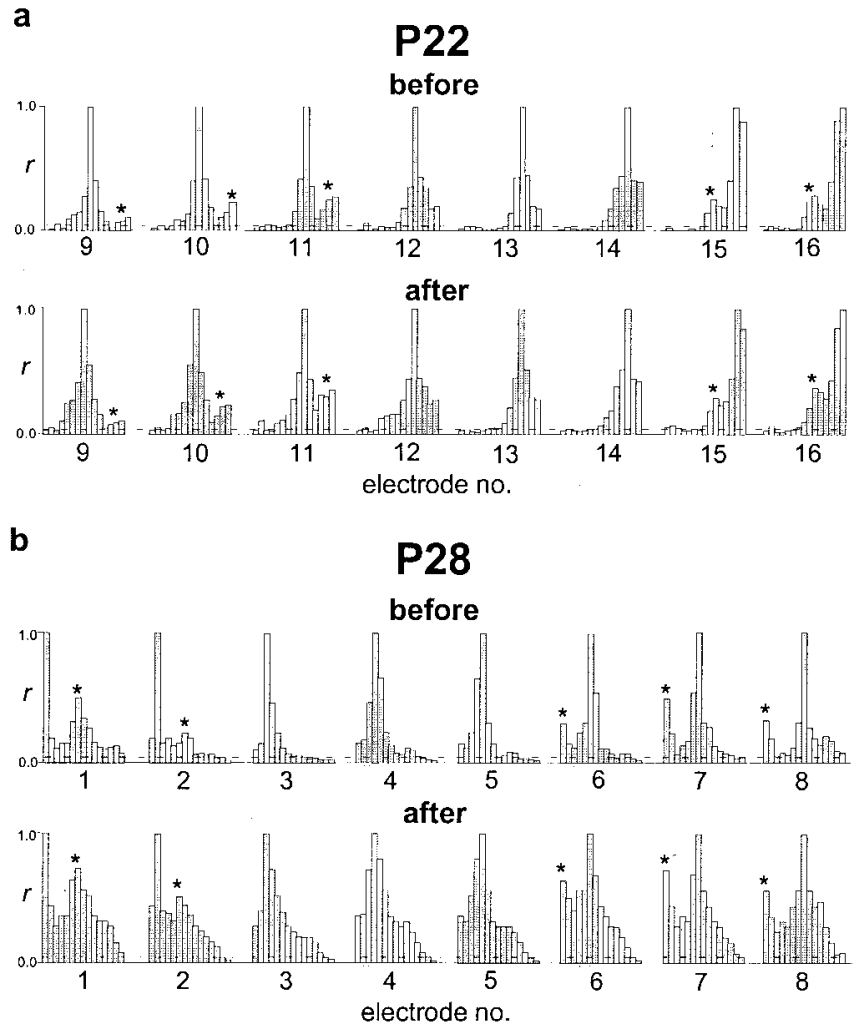

Figure 7. Correlated patterns of activity in the visual cortex remain stable despite the absence of LGN input activity. $a$, Top trace, Control cross-correlation maps in a P22 ferret over a single recording block before transiently blocking LGN activity by transection of both optic nerves. Bottom trace, Cross-correlation maps obtained in the same animal and at the same recording depth. Recordings were made within the first $45 \mathrm{~min}$ of cutting both optic nerves during which LGN activity was abolished. These maps show the same pattern of long-range secondary peaks. In each map, electrode 2 is the leftmost vertical bar, and electrodes 3-16 are successive bars to the right. Reciprocal long-range secondary peaks, marked by asterisks, are present at the same locations before and after transiently blocking LGN activity by cutting the optic nerves. The horizontal dash line $(r=0.04)$ shows the statistical significance level for computed correlation coefficients $(p<0.05) . b$, Spatial correlation maps from a single P28 ferret for electrodes 1-15. Control maps are shown in the top trace, and test correlation maps obtained in the same animal after cutting both optic nerves are shown in the bottom trace. Recording procedure was the same as described in $a$.

to be small and patchy, those in more dorsal parts of area 17 are irregular and large. In addition, there are striking inter-individual differences in the pattern of ocular dominance columns within comparable area 17 regions. This lack of uniformity in the pattern of ocular dominance bands in the ferret complicates the task of relating periodicity of patches of correlated activity to that of ocular dominance columns.

\section{Contribution of clustered horizontal connections}

A second potential mechanism underlying the patchy distribution of correlated activity is the system of clustered horizontal connections within layer 2/3 of area 17. Durack and Katz (1996) demonstrate that the initial emergence of crudely clustered horizontal connections begins at P28; however, one of two P24 ferrets in Ruthazer and Stryker (1996) also exhibited crudely clustered connections. Although the precise date of the emergence of clustered horizontal connections is not clear, the close 
timing of this process after the observation of long-range correlated activity at P22 suggests that these two events may be intimately related. It is possible that these patterns of correlated activity may reflect initial changes in functional synaptic connections that precede subsequent anatomical axon remodeling.

The patchy distribution of correlated activity within area 17 could provide a mechanism for the development and stabilization of clustered horizontal connections. Coincident firing between presynaptic axons originating from cells located at one cortical site, and postsynaptic cells located at a second coactive site, could result in the selective strengthening of these synapses and the growth and elaboration of local axon collaterals (Hebb, 1949). There is strong experimental evidence demonstrating that correlated activity plays an important role in shaping clustered lateral connections. In strabismus experiments that decorrelate activity between the two eyes, the pattern of clustered horizontal connections is altered such that horizontal connections selectively interconnect ocular dominance columns of the same eye, whereas in normal animals, there is no eye-specific selectivity (Löwel and Singer, 1992). Moreover, previous work has indicated that cortical activity is essential for the process of axonal remodeling by revealing that blocking spontaneous activity in the cortex with TTX is sufficient to prevent the formation of clustered lateral connections (Ruthazer and Stryker, 1996).

It is possible that, in some animals, the patches of correlated spontaneous activity observed at P22 may not reflect nascent clustered horizontal connections in area 17 but may reflect the mature clustered horizontal connections already present between areas 17 and 18 at this age (Ruthazer and Stryker, 1996). However, because the 17/18 border lies along the dorsal surface of the lateral gyrus (Law et al., 1988), we were careful to always place the electrode array mediolaterally and posterior to this location along the caudal bank of the lateral gyrus. This ensured that all electrodes would lie exclusively within area 17 posterior to the $17 / 18$ border. However, the exact location of the border can shift from animal to animal. Therefore, we cannot rule out the possibility that, in some animals, our recordings may have reflected the clustered horizontal connections already present between areas 17 and 18. Future experiments are necessary to determine the relationship between the patches of cortical correlated activity and the anatomically clustered horizontal connections.

\section{Contribution of retinal input to cortical burst patterns}

The presence of retinal waves before natural eye opening raises the possibility that retinal input is critical in the development of the visual system. It was shown that retinal waves are faithfully relayed to the LGN in vitro (Mooney et al., 1996). However, Weliky and Katz (1999) revealed that in vivo LGN activity cannot be predicted solely from patterns of retinal input activity. This work demonstrated the presence of binocular correlations between different eye-specific LGN layers that would not be expected if each eye bursts independently. In addition, after binocular enucleation, the LGN continues to burst in a manner that preserves the differential correlation in activity between different eye-specific layers. However, with the additional removal of cortical feedback to the LGN, this differentially correlated bursting activity is abolished. This indicates the prominent role of the thalamocortical loop in sustaining the correlational structure of LGN activity after the removal of retinal input activity. Our result demonstrating the persistence of the long-range pattern of correlated activity in the absence of retinal input provides additional support for a diminished role for retinal input in establishing activity patterns in higher stages of the visual system.

Consistent with this hypothesis, recent studies show that retinal input is neither important in the segregation of LGN afferents (Crowley and Katz, 1999) nor in the formation of clustered horizontal connections (Ruthazer and Stryker, 1996); binocular enucleation does not disrupt either of these two processes. Moreover, manipulations that generate unbalanced retinal input such as monocular enucleation does not affect the formation of early ocular dominance columns (Crowley and Katz, 2000). Although Crowley and Katz $(1999,2000)$ propose that molecular cues are responsible for the establishment of ocular dominance columns, another equally plausible explanation is that the segregation of LGN afferents is driven predominately by activity within the intact thalamocortical loop. In support of this possibility, Weliky and Katz (1999) show that, within the LGN, the eye-specific differences in correlated activity are maintained after binocular eye enucleation. Furthermore, the pattern of long-range correlated activity that persists in the visual cortex after transection of both optic nerves may provide cues to stabilize the clustered horizontal connections and segregated LGN afferents in the bilaterally enucleated animals.

Although retinal input activity is neither critical for the segregation of LGN afferents nor for the formation of clustered horizontal connections, we find that the temporal properties of cortical bursts are significantly altered after removal of retinal input. Together with previous work demonstrating that LGN bursting is similarly affected after transection of both optic nerves (Weliky and Katz, 1999), these results indicate that retinal activity plays an important role in establishing the timing of activity within the thalamocortical loop but not in determining the spatial pattern of that activity.

\section{REFERENCES}

Caríc D, Price DJ (1999) Evidence that the lateral geniculate nucleus regulates the normal development of visual corticocortical projections in the cat. Exp Neurol 156:353-362.

Chapman B, Stryker MP (1993) Development of orientation selectivity in ferret visual cortex and effects of deprivation. J Neurosci 13:5252-5262.

Chapman B, Stryker MP, Bonhoeffer T (1996) Development of orientation preference maps in ferret primary visual cortex. J Neurosci 16: 6443-6453.

Crowley JC, Katz LC (1999) Development of ocular dominance columns in the absence of retinal input. Nat Neuroscience 2:1125-1129.

Crowley JC, Katz LC (2000) Early development of ocular dominance columns. Science 290:1321-1324.

Durack JC, Katz LC (1996) Development of horizontal projections in layer $2 / 3$ of ferret visual cortex. Cereb Cortex 6:178-183.

Hebb DO (1949) The organization of behavior: a neuropsychological theory. New York: Wiley.

Katz LC, Shatz CJ (1996) Synaptic activity and the construction of cortical circuits. Science 274:1133-1138.

Law MI, Zahs KR, Stryker MP (1988) Organization of primary visual cortex (area 17) in the ferret. J Comp Neurol 278:157-180.

Löwel S, Singer W (1992) Selection of intrinsic horizontal connections in the visual cortex by correlated neuronal activity. Science 255:209-212.

Maffei L, Galli-Resta L (1990) Correlation in the discharges of neighboring rat retinal ganglion cells during prenatal life. Proc Natl Acad Sci USA 87:2861-2864.

Meister M, Wong ROL, Baylor DA, Shatz CJ (1991) Synchronous bursts of action potentials in ganglion cells of the developing mammalian retina. Science 252:939-943.

Miller R (1996) Neural assemblies and laminar interactions in the cerebral cortex. Biol Cybern 75:253-261.

Mooney R, Penn AA, Gallego R, Shatz CJ (1996) Thalamic relay of spontaneous retinal activity prior to vision. Neuron 17:863-874. 
Penn AA, Riquelme PA, Feller MB, Shatz CJ (1998) Competition in retinogeniculate patterning driven by spontaneous activity. Science 279:2108-2112.

Ruthazer ES, Stryker MP (1996) The role of activity in the development of long-range horizontal connections in area 17 of the ferret. J Neurosci 16:7253-7269.

Schwartz TH, Rabinowitz D, Unni V, Kumar VS, Smetters DK, Tsiola A, Yuste R (1998) Networks of coactive neurons in developing layer 1. Neuron 20:541-552.

Shatz CJ, Stryker MP (1988) Prenatal tetrodotoxin infusion blocks segregation of retinogeniculate afferents. Science 242:87-89.

Snedecor GW, Cochran WG (1989) Correlation. In: Statistical methods, Ed 8, pp 177-195. Ames, IA: Iowa State UP.
Weliky M, Katz LC (1999) Correlational structure of spontaneous neuronal activity in the developing lateral geniculate nucleus in vivo. Science 285:599-604.

White LE, Bosking WH, Williams SM, Fitzpatrick D (1999) Maps of central visual space in ferret V1 and V2 lack matching inputs from the two eyes. J Neurosci 19:7089-7099.

Wong ROL, Meister M, Shatz CJ (1993) Transient period of correlated bursting activity during development of the mammalian retina. Neuron 11:923-938.

Wong ROL, Chernjavsky A, Smith SJ, Shatz CJ (1995) Early functional neural networks in the developing retina. Nature 374:716-718.

Yuste R, Peinado A, Katz LC (1992) Neuronal domains in developing neocortex. Science 257:665-669. 